Research

\title{
A pilot study to assess the feasibility of evaluation of markers of response to chemotherapy at one day $\& 21$ days after first cycle of chemotherapy in carcinoma of breast: a prospective non-randomized observational study
} Shekhar Sharma*1, KR Hiran ${ }^{2}$, K Pavithran ${ }^{3}$ and DK Vijaykumar ${ }^{1}$

Address: ${ }^{1}$ Department of Surgical Oncology, Amrita Institute of Medical Sciences \& Research Center, Amrita Lane, Edapally, Ernakulam - 682026, Kerala, India, ${ }^{2}$ Department of Pathology, Amrita Institute of Medical Sciences \& Research Center, Amrita Lane, Edapally, Ernakulam - 682026, Kerala, India and ${ }^{3}$ Department of Medical Oncology, Amrita Institute of Medical Sciences \& Research Center, Amrita Lane, Edapally, Ernakulam - 682026, Kerala, India

Email: Shekhar Sharma* - drshekharsharma@gmail.com; KR Hiran - hiran_kr@rediffmail.com; K Pavithran - pavithrank@aims.amrita.edu; DK Vijaykumar - dkvijaykumar@aims.amrita.edu

* Corresponding author

Published: 30 March 2009

World Journal of Surgical Oncology 2009, 7:35 doi:10.1 186/1477-7819-7-35

This article is available from: http://www.wjso.com/content/7///35

(C) 2009 Sharma et al; licensee BioMed Central Ltd.

This is an Open Access article distributed under the terms of the Creative Commons Attribution License (http://creativecommons.org/licenses/by/2.0), which permits unrestricted use, distribution, and reproduction in any medium, provided the original work is properly cited.
Received: 10 December 2008

Accepted: 30 March 2009

\begin{abstract}
Background: Interest in translational studies aimed at investigating biologic markers in predicting response to primary chemotherapy (PCT) in breast cancer has progressively increased. We conducted a pilot study to evaluate feasibility of evaluating biomarkers of response to PCT at one \& 21 days after first cycle.

Methods: Adult, non-pregnant, non-lactating women with histologically confirmed infiltrating duct carcinoma underwent serial core biopsies after first cycle of PCT and these were scored for Ki-67, $\mathrm{BCl}-2$ and Caspase-3 using immunohistochemistry.

Results: We recruited 30 patients with a mean age of $5 \mathrm{I}$ years. We were successful $95.6 \%$ times in performing a core biopsy and of these $84.6 \%$ had adequate tissue in the cores harvested. After a mean of 4 cycles of PCT, 26 patients underwent surgery and good response was noted in 9 patients (30\%) using Miller-Payne criteria. There was a trend noted in all markers, which appeared different in those with good response and poor response. Good responders had significantly higher $\mathrm{Ki}-67$ and significantly lower $\mathrm{BCl}-2$ at baseline and a significant decrease in Ki-67 and Caspase-3 at 2 I days after the first chemotherapy.

Conclusion: We report a detectable change in biomarkers as early as 24-48 hours after the first chemotherapy along with a definite trend in change that can possibly be used to predict response to chemotherapy in an individual patient. The statistical significance and clinical utility of such changes needs to be evaluated and confirmed in larger trials.
\end{abstract}

\section{Background}

There is increasing interest in the ways and means to predict the response of an individual patient to primary chemotherapy (PCT) with an ultimate interest to predict individual responses to treatment in the minimum time feasible. 
Clinical response has been used as an intermediate, surrogate end-point for assessment of the efficacy of PCT in an individual, although this assessment is far from accurate [1]. Tools are, therefore, required to better assess the efficacy of chemotherapy regimen.

Ellis et al showed that chemotherapy induced apoptosis in early breast cancer could be demonstrated soon after the chemotherapy [2]. In continuation of this, it would indeed be useful to have a marker of response that can be evaluated as soon as possible after the first cycle of chemotherapy and correlates to the clinical outcome.

We wanted to evaluate if it was feasible to harvest a satisfactory core biopsy immediately after first cycle and just prior to second cycle of chemotherapy, when patient is available in the hospital along with feasibility to evaluate biomarkers of response to chemotherapy in these biopsies. The correlation to response, if proven, would help clinicians to tailor chemotherapy to individual patients and may provide the opportunity to offer earlier possible alternative, non-cross-resistant regimens to those patients not achieving a response to the initial regimen.

We proposed to explore the change in biomarkers of response to PCT at one day and 21 days after the first cycle of PCT in women with breast cancer attending our institution for care and towards this aim we initiated a pilot study in our institution, after appropriate scientific and ethics committee approval, in the patients of breast cancer undergoing PCT. The primary aims of this pilot study were to assess the feasibility and reproducibility of performing:

a) Serial core biopsies one day and 21 days after first cycle of chemotherapy, with emphasis on patient acceptance and complications of the procedure.

b) Assays of apoptosis (Caspase-3 \&Bcl-2) and proliferation index (Ki-67) in patients of carcinoma of breast on core biopsy specimens using immunohistochemistry (IHC).

c) Quantification of extent of change in these biomarkers of response to chemotherapy one day and 21 days after first cycle of chemotherapy.

Table I: Details of IHC antibodies for Caspase-3, Bcl-2 and Ki-67 d) Histopathological response grading at final surgical histopathology using Miller-Payne response assessment criteria.

\section{Patients and methods}

Adult (more than 18 years of age) non-pregnant, non-lactating women with histologically confirmed, previously untreated infiltrating duct carcinoma (IDC) of breast who were advised PCT, as per institutional protocol, were eligible. Patients with inflammatory breast cancer or those with history of any indigenous form of therapy for breast cancer were excluded from this study. The study was approved by the Institute review board.

After an informed written consent, serial core biopsies were taken before (C0 biopsy), 24-48 hours (C1 biopsy) and 21 days (C2 biopsy) after first cycle of chemotherapy. Chemotherapy regimen was at discretion of the treating medical oncologist. Serial core biopsies were obtained exclusively for the purpose of this study for determination of potential predictive surrogate markers of response. A core biopsy was obtained using Bard Monopty disposable biopsy instrument (Covington, GA). Three core biopsies were taken - first before starting chemotherapy (C0), second 24-48 h after cycle one (C1), and third 21 days after cycle one (C2). Biopsy specimens, two cores each time, were fixed in $10 \%$ buffered formalin and embedded in paraffin and sectioned into $4 \mu \mathrm{m}$-thick sections.

Surgery was scheduled after completion of 2-6 cycles of PCT according to patient's response to chemotherapy and at discretion of the treating physicians. The study pathologist carefully evaluated the definitive surgical specimen for the presence of residual disease and grading of pathological response to chemotherapy was done using MillerPayne criteria for assessment of response to chemotherapy [3]. Miller-Payne response grade $4 \& 5$ were considered as good pathological response (GPR) while grades 1 to 3 were considered poor pathological response (PPR). ER, $P R$, and HER2/neu were evaluated only on C0 biopsy.

Markers for proliferation (Ki-67), Caspase-3 and Bcl-2 were evaluated by IHC using appropriate antibodies (Table 1). Slides were deparaffinized and hydrated. Standard techniques for antigen retrieval, blocking endogenous peroxi-

\begin{tabular}{llll}
\hline Antigen & Antibody & Manufacturer & Scoring \\
\hline Ki-67 & MAb & Zymed, San Francisco, CA & Nuclear staining; \% positive \\
\hline $\mathrm{BCl}-2$ & MAb & DAKO, Carpenteria, CA & Cytoplasmic staining, \% positive \\
\hline Caspase-3 & Mouse & Imgenex, San diego, CA & Nuclear and cytoplasm staining, \% positive \\
\hline
\end{tabular}

MAb - Monoclonal antibody 
dase activity and nonspecific antibody binding were followed before immuno-staining with commercially available antibodies (Table 1). Primary antibodies were pre-diluted except for Caspase-3 for which a dilution of 1:500 was used. Incubation period for all the antibodies were 1 hour except Ki-67 that was kept for 2 hours at $37^{\circ} \mathrm{C}$. Known positive and negative controls were included for each batch run. Slides were scored for percentage of positive cells and relative intensity.

The feasibility of performing serial core biopsies was not addressed statistically. Non-parametric tests were applied to assess the other variables. Patient baseline characteristics, the treatment regimen, and molecular markers were each assessed for an association with pathologic response using the Mann-Whitney $U$ test. The change in biomarkers of response from pre-treatment was assessed in the GPR \& PPR groups by paired comparisons, using the Wilcoxon signed rank test, while within group analysis was performed using Wilcoxon rank sum test.

\section{Results}

We recruited 30 patients of breast cancer with a mean age of 51 years $( \pm 8.4)$ for this study from April 2007 to June 2008.

Patient demographics are mentioned in Table 2. Disease characteristics are mentioned in Table 3 . There were no significant differences in demographic pattern between GPR \& PPR groups.

ER, PR and Her-2/neu receptors were all positive in four $(13.3 \%)$ patients while all three were negative in 10 (33.3\%) patients. Of the 10 patients (33.3\%) with Stage IV disease, whole body skeletal scintigraphy detected metastasis in eight patients (26.7\%); chest X-ray in one patient (3.3\%); ultrasound abdomen in four patients $(13.3 \%)$ and CT scan chest in two patients (6.7\%).

We were successful in harvesting core biopsy tissue with adequate cellularity in a reasonable proportion of patients (Table 4). Of the proposed 90 core biopsy procedures (three each in 30 patients), only four patients $(4.44 \%)$ refused the third core biopsy (C2) due to procedure related pain. We did not observe any other procedure related complications.

Although paucicellular harvest can be attributed to poor technique and less number of cores taken, it is interesting to note that out of the four patients who had a paucicellular harvest at 21 days after chemotherapy (C2 biopsy), three had a good response on final histopathology by Miller-Payne criteria.

Chemotherapy regimens included Adriamycin \& Cyclophosphamide followed by Paclitaxel $(\mathrm{AC}+\mathrm{T})$ in 12
Table 2: Patient demographics

\begin{tabular}{|c|c|c|}
\hline Characteristic & Mean ( \pm SD) & Range \\
\hline Age (years) & $51( \pm 8.4)$ & $31-63$ \\
\hline Duration of symptoms (months) & $10.54( \pm 10.88)$ & $0.25-40$ \\
\hline Age at Menarche (years) & $14.16( \pm 1.7)$ & $11-18$ \\
\hline Age at Marriage (years) & $21.58( \pm 4.26)$ & $13-33$ \\
\hline Age at menopause (years) & $47.58( \pm 3.73)$ & $4 I-54$ \\
\hline Parity (median) & 2 & $0-5$ \\
\hline Age at first childbirth & $23.81( \pm 4)$ & $17-32$ \\
\hline Duration of breast feeding (months) & $43.22( \pm 22.02)$ & $6-96$ \\
\hline Number of PCT cycles (median) & 4 & $3-9$ \\
\hline \multicolumn{3}{|l|}{ Menstrual status } \\
\hline Premenopausal & 19 & 63.3 \\
\hline Postmenopausal & 11 & 36.7 \\
\hline \multicolumn{3}{|l|}{ Laterality } \\
\hline Right & 18 & 60 \\
\hline Left & 11 & 36.7 \\
\hline Bilateral & 1 & 3.3 \\
\hline
\end{tabular}

patients (40\%); combination of Docetaxel, Adriamycin and Cyclophosphamide (TAC) in 10 patients (33.3\%); 5Flurouracil, Adriamycin (or Epirubicin) and Cyclophosphamide (FAC/FEC) in 6 patients $(20 \%)$ and Docetaxel alone in 2 patients $(6.7 \%)$

Miller-Payne pathological response category could not be assessed in 4 patients (13.3\%) (one expired, one had progressive disease on chemotherapy, one refused surgery and one had surgery cancelled due to chemotherapy induced cardiomyopathy). Details of Miller-Payne pathological response in the remaining 26 patients are shown in Table 3. Nine patients (30\%) had a GPR to chemotherapy (Table 3).

Levels of Ki-67, $\mathrm{Bcl}-2$, and Caspase-3 and their comparisons in C0, C1 and C2 biopsy are shown in Figures 1, 2 and 3 respectively.

We observed that GPR group had significantly higher Ki67 at baseline $(\mathrm{p}=0.042)$ and both GPR \& PPR groups 
Table 3: Disease characteristics

\begin{tabular}{|c|c|c|c|}
\hline \multicolumn{2}{|l|}{ Parameter } & $\mathbf{N}$ & Percent \\
\hline \multicolumn{4}{|c|}{ Stage of disease at presentation } \\
\hline \multicolumn{2}{|l|}{ II $B$} & 4 & 13.3 \\
\hline \multicolumn{2}{|l|}{ III A } & 7 & 23.3 \\
\hline \multicolumn{2}{|l|}{ III B } & 5 & 16.7 \\
\hline \multicolumn{2}{|l|}{ III C } & 4 & 13.3 \\
\hline \multicolumn{2}{|l|}{ IV } & 10 & 33.3 \\
\hline \multicolumn{4}{|l|}{ Histological type } \\
\hline \multicolumn{2}{|c|}{ Infiltrating ductal carcinoma (IDC) } & 27 & 90.0 \\
\hline \multicolumn{2}{|c|}{ Infiltrating lobular carcinoma (ILC) } & 2 & 6.7 \\
\hline \multicolumn{2}{|l|}{ Combined ILC \& IDC } & 1 & 3.3 \\
\hline \multicolumn{4}{|l|}{ Grade of tumor } \\
\hline \multicolumn{2}{|l|}{ Low grade } & 4 & 13.3 \\
\hline \multicolumn{2}{|l|}{ Intermediate grade } & 14 & 46.7 \\
\hline \multicolumn{2}{|l|}{ High grade } & 12 & 40.0 \\
\hline \multicolumn{4}{|c|}{ Miller-Payne response \# } \\
\hline Poor & Grade I (no or $<10 \%$ response) & 5 & 16.7 \\
\hline pathological & Grade II (I0-30\% response) & 4 & 13.3 \\
\hline response (PPR) & Grade III (30-90\% response) & 8 & 26.6 \\
\hline Good pathological & Grade IV (>90\% response) & 4 & 13.3 \\
\hline response (GPR) & Grade $V$ (complete response or few isolated tumor cell islands remaining) & 5 & 16.7 \\
\hline
\end{tabular}

\#: Miller Payne response could not be assessed in 4 patients who did not undergo surgery

showed a rise at 24-48 hours after first chemotherapy (in C1 biopsy). This decreased 21 days after first chemotherapy to below the baseline values (in GPR group) as well as $\mathrm{C} 2$ values in PPR group, which were static at $\mathrm{C} 1$ levels. The difference between GPR \& PPR groups in levels of Ki-67 seen in C2 biopsy was not significant ( $p=n s)$, although the difference in change from $\mathrm{C} 1$ to $\mathrm{C} 2$ appears striking with a steep slope in GPR group (Figure 1c).

On the converse, $\mathrm{Bcl}-2$ was significantly lower in GPR group in all the three biopsies $(\mathrm{p}=0.015 ; 0.014 ; 0.039$ for
C0, C1 \& C2 respectively). Chemotherapy induced a steady rise in the entire group, which was steeper in GPR group from $\mathrm{C} 1$ to $\mathrm{C} 2$. Bcl-2 peaked at biopsy taken at 2448 hours after the first cycle in the PPR group and then had a plateau to nearly same level at 21 days (Figure 2c).

Caspase-3 values peaked at 24-48 hours before falling to near baseline levels at 21 days after the first chemotherapy with nearly similar baseline and peak values in both the groups ( $\mathrm{p}=\mathrm{ns}$ for both $\mathrm{C} 0$ \& $\mathrm{C} 1$ biopsies). The decline in GPR group for values of Caspase-3 from C1 to C2 biopsy 
Table 4: Feasibility \& adequacy of core biopsy procedures

\begin{tabular}{|c|c|c|c|}
\hline Procedure & & $\mathbf{N}$ & $\%$ \\
\hline Core biopsy at baseline (C0 biopsy) & & 30 & 100 \\
\hline Core biopsy $24-48$ hours after first cycle of chemotherapy ( $\mathrm{Cl}$ biopsy) & & 30 & 100 \\
\hline Core biopsy $2 \mathrm{I}$ days after first cycle of chemotherapy ( $\mathrm{C} 2$ biopsy) & & 26 & 86.6 \\
\hline \multicolumn{4}{|l|}{ Adequacy } \\
\hline & At C0 biopsy & 30 & 100 \\
\hline & At Cl biopsy & 28 & 93.3 \\
\hline & At C2 biopsy & 22 & $73.3(84.3 \%$ of 26 attempted $)$ \\
\hline \multicolumn{4}{|l|}{$4(13.3 \%)$ refused $C 2$ biopsy; $4(13.3 \%)$ had paucicellular harvest on $C 2$ biopsy } \\
\hline Definitive surgery & & 26 & 86.6 \\
\hline
\end{tabular}

4 (13.3\%) patients did not undergo surgery due to different reasons.

was steeper, the difference from PPR group being significant $(\mathrm{p}=0.024)$ at this point. In the GPR group, the Caspase-3 values at $\mathrm{C} 2$ fell below the baseline values (C0) (Figure 3c).

In our study, the magnitude of change in $B c l-2$ within 2448 hours after the first chemotherapy, in the entire group, was significant ( $\mathrm{p}=0.04)$, while that for markers of proliferation (Ki-67) and apoptosis (Caspase-3) was not significant $(\mathrm{p}=\mathrm{ns})$.

Thus, tumors with a higher Ki- 67 at baseline along with a low $\mathrm{Bcl}$-2 (anti-apoptotic gene) responded better to chemotherapy. In other words, high rates of apoptosis and proliferation at baseline were associated with improved pathological response. Another interesting observation during this study was that at 21 days, a decrease in Ki-67 and Caspase -3 was predictive of favorable response $(\mathrm{p}=$ 0.01 for both).

In this study, ER-positive tumors had a significant association with poor response $(\mathrm{p}=0.014)$ and had a higher $B c l$ 2 expression at baseline (mean $B c l$-2 35.38 in ER-positive vs. mean $\mathrm{Bcl}-214.35$ in ER negative tumors; $\mathrm{p}=0.04$ ). There was no difference in expression of Ki-67 or Caspase3 in ER-positive or negative tumors or in expression of these markers or in response between $P R$ and Her2/neu positive or negative tumors.

As a word of caution, $\mathrm{p}$ values of significance should be interpreted with caution due to the small sample size. It was primarily aimed as a pilot study to verify feasibility and reproducibility of this trial design and to see if changes in biomarkers could be measured and quantified at patient-friendly time points, aims that it apparently has achieved.

We faced problems using Caspase-3 to evaluate the apoptotic index, as this terminal enzyme of the apoptotic cascade is cytoplasmic in location. This led to a diffuse staining of slides, which caused difficulty in interpretation of positive cells and percentage positivity. Additionally, technical expertise in slide preparation and IHC staining were other major hurdles in the initial phase of the study.

On the basis of this pilot study, we observe that this trial design is feasible (in this context, patient acceptable without any specific objective incentive) and quantification of biomarkers of response to chemotherapy can be performed on these core biopsies. There is a trend towards change noted in these markers (in this study, Ki-67, Bcl-2, Caspase-3) both at 24 hours and at 21 days after the first cycle of chemotherapy, although these results need to be confirmed in larger studies. In our experience, MillerPayne criteria to assess response to chemotherapy, is an easily reproducible method of grading response objectively.

We hope that we will be able to improve the adequacy of tissue by increasing the number of cores harvested each time from two in the present study to three or four in future studies. A more proactive approach to pain medication prescription will, hopefully, help us in preventing dropouts in further trials. However, we would need alter- 

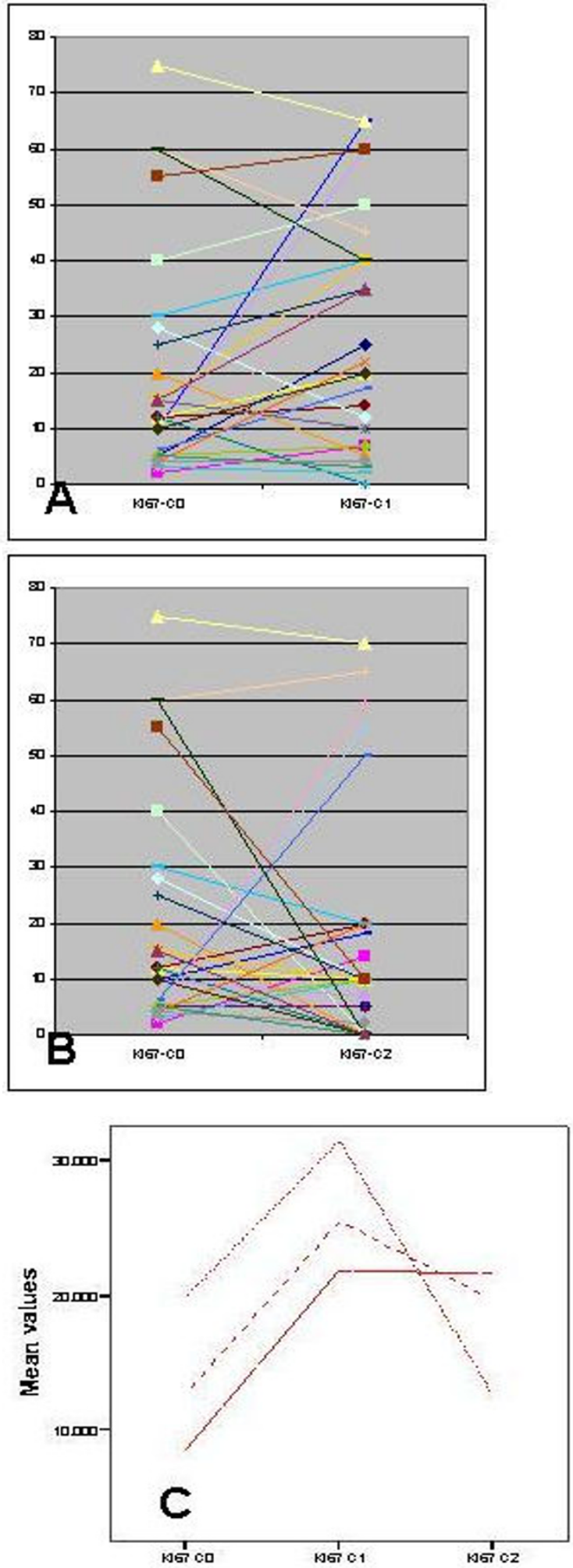

\section{Two response}

_ poor responders

......... Good responders

- . - - Entire group

Figure I

Comparison of Ki-67 levels in (a) C0-CI biopsy; and (b) C0-C2 biopsy; and (c) Change in mean value over time. 

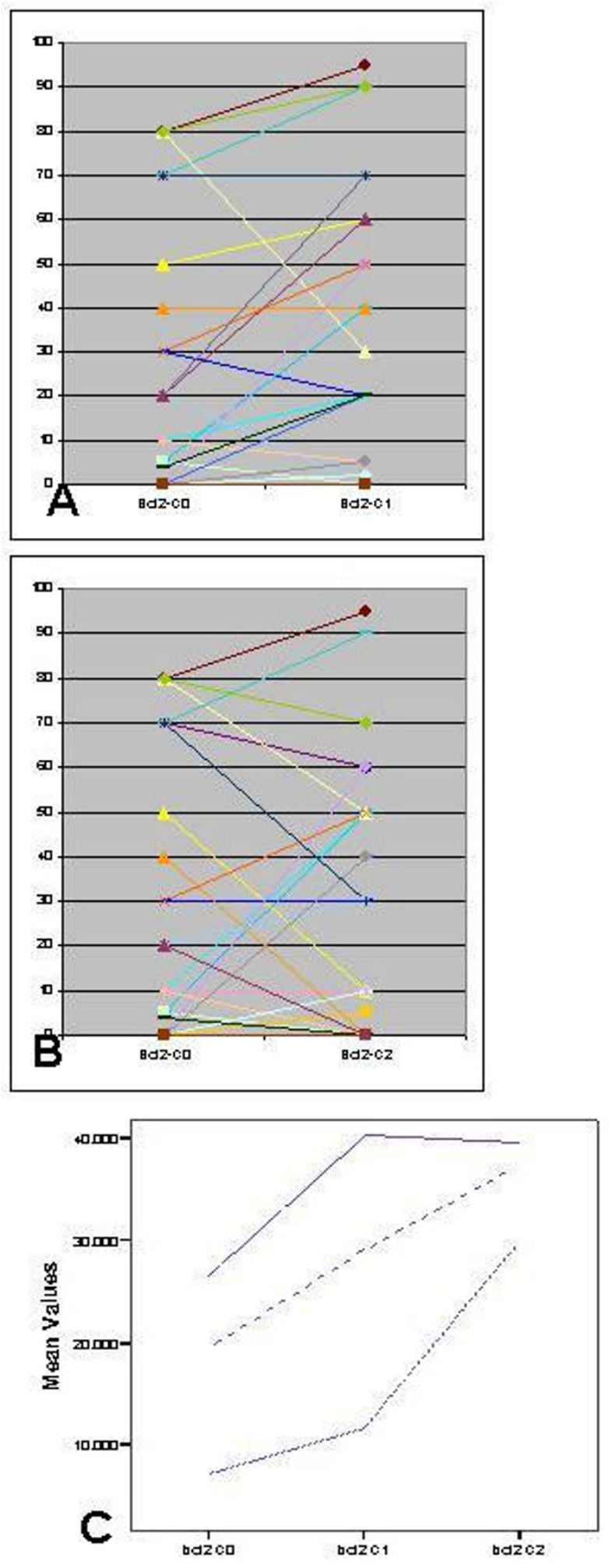

Figure 2

Comparison of Bcl-2 levels in (a) C0-Cl biopsy; and (b) C0-C2 biopsy; and (c) Change in mean value over time. 

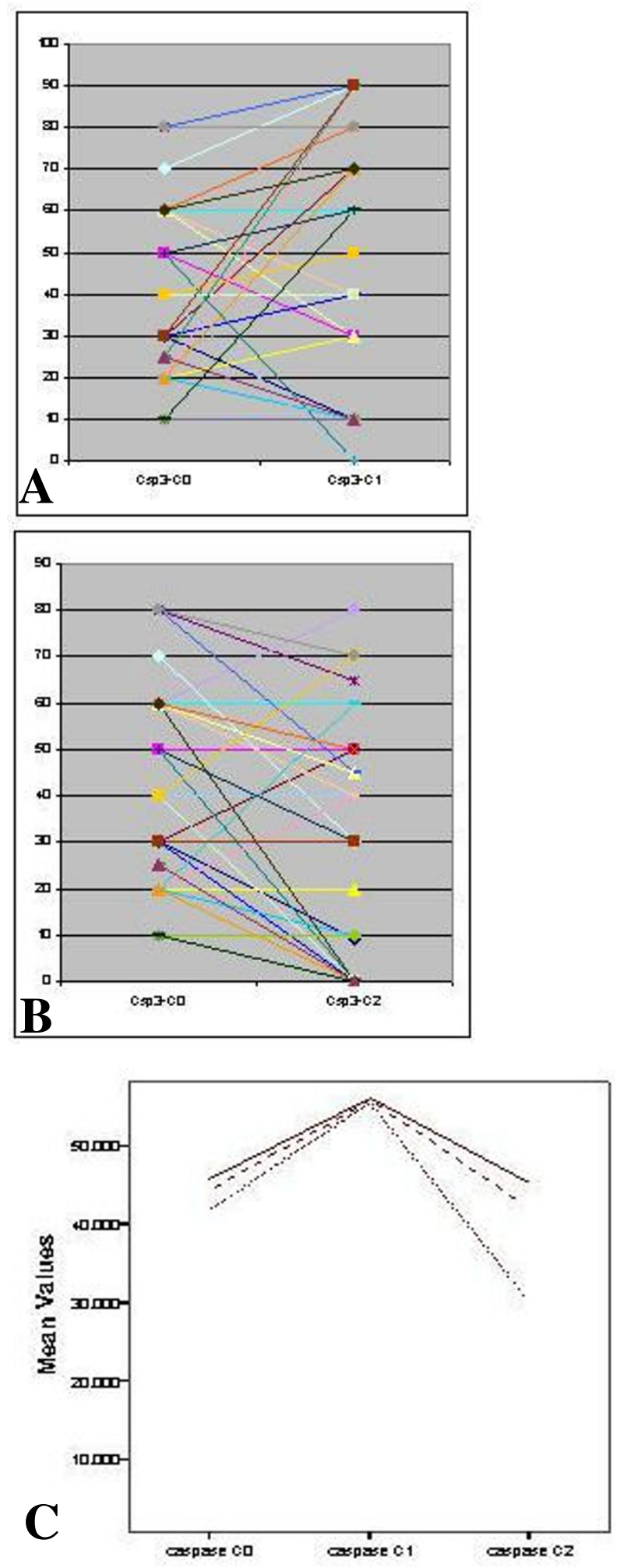

Two response - poor responders -....... Good respoide rs - - - Eutire group

Figure 3

Comparison of Caspase-3 (Csp-3) levels in (a) C0-CI biopsy; and (b) C0-C2 biopsy; and (c) Change in mean value over time. 
native methods of evaluating apoptotic index (eg TUNEL, etc) due to problems associated with Caspase-3 in any future study.

Whether we need to repeat the same design (two biopsies after baseline - one 24-48 hours and second 21 days after first chemotherapy) or either one of these biopsies can be omitted is a matter of debate, although in our opinion a three point measurement will improve the predictive power of the larger trial.

\section{Discussion}

Pathological complete response to PCT has been correlated with long-term outcome [4,5], although this is seen in only $3-30 \%$ of patients [6]. Bio-molecular predictors of tumor response to primary CT include S-phase fraction, $\mathrm{ER}, \mathrm{PgR}$, thymidine labeling index, ploidy, p53 and cerbB-2 (Her-2/neu) [7-12].

There is preliminary evidence that supports proliferation \& apoptosis-related markers as predictors of long-term response to PCT $[13,14]$. These include, among others, markers for induction of apoptosis, expression of $\mathrm{Bcl}-2$, and proliferation index (Ki-67 assay) [2,15,16]. However the exact relationship of the levels of biomarkers in a tumor in pre and post chemotherapy setting is relatively under-explored.

Studies have usually evaluated markers for response to chemotherapy after a significant delay [2]. A time gap of 10 days or more poses a difficult hurdle for investigators to have the patient come back again for tissue harvesting alone with most patients being reluctant to do so in absence of any objective incentive for their extra time, effort and expenses. This is a more acute issue in the Indian perspective, where patients often need to travel great distances to seek medical care.

We chose to evaluate three biomarkers, namely Ki-67 (marker of proliferation), Bcl-2 and Caspase-3 (anti- and pro-apoptotic markers) as data exist showing a close relationship between apoptosis and proliferation in untreated tumours $[17,18]$. The decision to restrict the number of biomarkers to three was to keep the study design as simple as possible in the pilot trial.

Several groups have found that Ki-67 decreases after chemotherapy over a variable duration [19]. Some studies have demonstrated a relationship of change in Ki-67 with response $[15,20]$. In a similar pilot study where Ki-67 was measured in 20 patients treated with chemo-endocrine therapy (mitoxantrone, mitomycin $\mathrm{C}$, methotrexate and tamoxifen), a decrease at day 10 or 21 after the first course of treatment correlated with response at 3 months $(p=$ $0.008)$. Ki-67 changes between the responders and non- responders were significant for both absolute and percentage change in the chemotherapy ( $p=0.01$ and $p=0.005$, respectively) as well as in chemo-endocrine therapy group ( $p=0.03$ and $p=0.06$, respectively) [21]. Further follow up showed that this decrease in Ki-67 after 10-21 days of therapy had a significant association with good clinical response on univariate analysis [15]. While significant associations with response have been revealed in these studies, none have assessed the predictive power in individual patients.

Whilst some studies have shown that a high proliferative index is a poor prognostic indicator $[22,23]$, others have debated this with observations that patients with highly proliferative tumours respond well to chemotherapy [24]. Honkoop et al showed that a high proliferative index in residual tumours after neoadjuvant chemotherapy and endocrine therapy was associated with a decreased disease free survival [25].

In this study, we noted that a higher baseline Ki- 67 was associated with better response to chemotherapy, probably because a higher fraction of these proliferative tumors at initiation of chemotherapy were susceptible to chemotoxic effects. The low 21-day Ki-67 values, in good responders, similar to those reported in literature, are indirect evidence of the efficacy of the chemotherapy in these patients in eliminating the mitotic fraction. It is intriguing to note that, as soon as 24 hours after chemotherapy, there was a rise in $\mathrm{Ki}-67$ levels, something that, to our knowledge, has not been reported in literature.

$\mathrm{Bcl}-2$ gene encodes for a $26-\mathrm{kDa}$ protein that mainly inhibits apoptosis. However, the role of $\mathrm{Bcl}-2$ expression on clinical outcome following chemotherapy is still under investigation, since available data are in some instances contrasting [26]. Also, interpretation of treatment benefit as a function of biomarkers is difficult in the absence of randomized, controlled trials.

A number of studies, covering about 5000 patients, with breast cancer at different stages showed that $\mathrm{Bcl}-2$ overexpression correlated to a differentiated phenotype and a favorable prognosis in patients subjected to localregional, hormonal or cytotoxic therapies $[14,27]$.

Our data suggests that breast carcinomas with low baseline apoptosis may respond poorly to chemotherapy. We observed a significant inverse correlation between expression of $\mathrm{Bcl}-2$ and response to the chemotherapy. These results are in general line with the postulated anti-apoptotic function of $\mathrm{Bcl}-2$ gene, higher levels in poor responders indicating a possible immunity from chemotherapy induced apoptosis. 
Some possible explanations for these paradoxical results have been mentioned in literature and include a complex interaction of $\mathrm{p} 53$ or its mutant variations with $\mathrm{Bcl}-2$, an inhibitory effect of $\mathrm{Bcl}-2$ on proliferation along with regulation of $\mathrm{Bcl}-2$ expression by estrogen and presence of antagonists, which may negate its anti-apoptotic function $[13,28]$.

The prognostic and predictive value of apoptotic markers in breast cancer is not yet fully understood. There is some suggestion that apoptotic index is an independent prognostic factor. Our results are similar to other reports in the literature that chemotherapy induces early changes in apoptosis [2].

Data from this study and another similar study [29] suggest that it may be possible, in future, to determine, as early as $24-48 \mathrm{~h}$ after administration of chemotherapy, whether a woman is likely to respond to a specific agent or not, information that might help to make an early decision regarding any change in such treatment. The novel approach in this study can also answer questions regarding the role of other markers and response to individual therapies.

This study does have a few limitations like small sample size (30 patients were recruited as this was planned as a pilot study only), heterogeneous patient population (no stratification on the basis of receptor status, chemotherapeutic regimen received or stage of disease) all of which in themselves can argue for a different disease biology and consequently difference in responses to chemotherapy. However, even with these limitations, results are impressive enough to favor larger, more rigorously controlled trials to confirm these.

\section{Conclusion}

In summary, we present a clinical design incorporating sequential core biopsy after first cycle of PCT in breast cancer that can be used as a model in future trials to correlate surrogate end point biomarkers with response. The model can also be used to incorporate novel agents with standard treatments. Changes in biomarkers like apoptosis and proliferation can then, if validated with larger trials using standard regimens, be used to determine the efficacy and/ or superiority of the novel combinations compared to standard treatments.

Whether or not trends observed in this study are significant and whether these can be used to tailor chemotherapy (our ultimate aim) awaits larger trials. Further studies, including a larger sample size receiving single standardized chemotherapy regimen, are warranted, especially in a prospective manner with uniform methods of measurement and cut-off points to assess the potential value of molecular markers in clinical practice. These studies will need to include multiple assays such as nuclear grade, levels of expression of p53, markers for cell proliferation, multi-drug resistance, and apoptosis [30].

\section{Competing interests}

The authors declare that they have no competing interests.

\section{Authors' contributions}

SS was instrumental in design the concept, patient recruitment, data analysis, manuscript preparation and editing. HKR was instrumental in designing the trial, evaluation of slides for data generation, manuscript preparation and editing. PK, and DKV were instrumental in ratifying study design, patient recruitment, literature search and manuscript editing \& final approval. All authors accept the responsibility of contents of this manuscript.

\section{Acknowledgements}

We wish to acknowledge the funding support for this study from Kerala State Council for Science, Technology \& Environment, Government of Kerala, India. Authors declare that the funding agency was not involved in the trial at any stage starting from concept to analysis and its role was limited to providing grant to conduct the research work. Additionally, we also wish to acknowledge the support of Ms Smitha, Lecturer, Dept of Biostatistics, AIMS, Cochin in analysis of the data.

\section{References}

I. Abu-Farsakh H, Sneige N, Atkinson EN, Hortobagyi G: Pathologic Predictors of Tumor Response to Pre-operative Chemotherapy in Locally Advanced Breast Cancer. Breast 1995, I:96-I0I.

2. Ellis PA, Smith IE, McCarthy K, Detre S, Salter J, Dowsett M: Preoperative chemotherapy induces apoptosis in early breast cancer. Lancet 1997, 349:849.

3. Ogston KN, Miller ID, Payne S, Hutcheon AW, Sarkar TK, Smith I, Schofield A, Heys SD: A new histological grading system to assess response of breast cancers to primary chemotherapy: Prognostic significance and survival. Breast 2003, I 2:320-327.

4. Kuerer HM, Newman LA, Smith TL, Ames FC, Hunt KK, Dhingra K, Theriault RL, Singh G, Binkley SM, Sneige N, Buchholz TA, Ross MI, Mcneese MD, Buzdar AU, Hortobagyi GN, Singletary SE: Clinical course of breast cancer patients with complete pathologic primary tumor and axillary lymph node response to doxorubicin-based neoadjuvant chemotherapy. J Clin Oncol 1999. I7:460-469.

5. Jones RL, Smith ID: Neoadjuvant treatment for early-stage breast cancer: opportunities to assess tumor response. Lancet Oncology 2006, 7:869-74.

6. Wolmark N, Wang J, Mamounas E, Bryant J, Fisher B: Preoperative chemotherapy in patients with operable breast cancer: nineyear results from national surgical adjuvant breast and bowel project B-1 8. J Natl Cancer Inst Monogr 2001:96-102.

7. Valero V, Buzdar AU, Hortobagyi GN: Locally advanced Breast Cancer. Oncologist 1996, I:8-17.

8. Resnick JM, Sneige N, Kemp BL, Sahin A, Ordonez NG, Frye DK, Hortobagyi GN: p53 and c-erbB-2 expression and response to preoperative chemotherapy in locally advanced breast carcinoma. Breast Disease 1995, 8b: |49-158.

9. Wood WC, Budman DR, Korzun AH, Cooper MR, Younger J, Hart JD, Moore A, Ellerton JA, Norton L, Ferree CR, Ballow AC, Henderson IC: Dose and dose intensity of adjuvant chemotherapy for stage II, node-positive breast carcinoma. N Engl J Med 1994, 330( (18): I 253-I 259.

10. Hortobagyi GN, Buzdar AU: Locally advanced breast cancer: a review including the M.D. Anderson experience. In High-Risk 
Breast Cancer Therapy Edited by: Ragaz J, Ariel IM. Berlin: SpringerVerlag; |991:382-415

II. McCready DR, Hortobagyi GN, Kau SW, Smith TL, Buzdar AU, Balch CM: The prognostic significance of lymph node metastases after preoperative chemotherapy for locally advanced breast cancer. Arch Surg 1989, I 24(I):2I-25.

12. Valagussa P, Zambetti M, Bonadonna G, Zucali R, Mezzanotte G, Veronesi U: Prognostic factors in locally advanced non-inflammatory breast cancer: long-term results following primary chemotherapy. Breast Cancer Res Treat 1990, I 5(3): I37-147.

13. Krajewski S, Blomvqvist C, Franssila K, Krajewska M, Wasenius V-M, Niskanen E, Reed JC: Reduced expression of pro-apoptotic gene $B a x$ is associated with poor response rates to combination chemotherapy and shorter survival in women with metastatic breast adenocarcinoma. Cancer Research 1995 , 55:447| -4478

14. Daidone MG, Luisi A, Veneroni S, Benini E, Silvestrini R: Clinical studies of $\mathrm{Bcl}-2$ and treatment benefit in breast cancer patients. Endocrine-Related Cancer 1999, 6:61-68.

15. Chang J, Powles TJ, Allred DC, Ashley SE, Clark GM, Makris A, Assersohn L, Gregory RK, Osborne CK, Dowsett : Biologic markers as predictors of clinical outcome from systemic therapy for primary operable breast cancer. J Clin Oncol 1999, 1 7:3058-3063.

16. Dowsett M, Archer C, Assersohn L, Gregory RK, Ellis PA, Salter J, Chang J, Mainwaring P, Boeddinghaus I, Johnston SR, Powles TJ, Smith IE: Clinical studies of apoptosis and proliferation in breast cancer. Endocr Relat Cancer 1999, 6:25-28.

17. Ellis PA, Smith IE, Detre S, Burton SA, Salter J, A'Hern R, Walsh G, Johnston SRD, Dowsett M: Reduced apoptosis and proliferation and increased $\mathrm{Bcl}-2$ in residual breast cancer following preoperative chemotherapy. Breast Cancer Res Treat 1998 48:107-116.

18. Lipponen P, Aaltomaa S, Kosma VM, Syrjanen K: Apoptosis in breast cancer as related to histopathological characteristics and prognosis. Eur J Cancer 1994, 30A:2068-2073.

19. Makris A, Powles T], Dowsett M, Osborne CK, Trott PA, Fernando IN, Ashley SE, Ormerod MG, Titley JC, Gregory RK, Allred DC: Prediction of response to neoadjuvant chemoendocrine therapy in primary breast carcinomas. Clin Cancer Res 1997, 3:593-600.

20. Makris A, Powles TJ, Allred DC, Ashley S, Ormerod MG, Titley JC, Dowsett $M$ : Changes in hormone receptors and proliferation markers in tamoxifen treated breast cancer patients and the relationship with response. Breast Cancer Res Treat 1998, 48: I I-20.

21. Makris A, Powles T], Allred DC, Ashley SE, Trott PA, Ormerod MG Titley JC, Dowsett M: Quantitative changes in cytological molecular markers during primary medical treatment of breast cancer: a pilot study. Breast Cancer Res Treat 1999, 53:5I-59.

22. Assersohn L, Salter J, Powles T], A'hern R, Makris A, Gregory RK, Chang J, Dowsett M: Studies of the potential utility of Ki67 as a predictive molecular marker of clinical response in primary breast cancer. Breast Cancer Res Treat 2003, 82: I |3-123.

23. Railo M, Lundin J, Haglund $C$, von Smitten K, von Boguslawsky K, Nordling S: Ki67, p53, Er-receptors, ploidy and S-phase as prognostic factors in $\mathrm{TI}$ node negative breast cancer. Acta Oncol 1997, 36:369-374.

24. Tewari M, Krishnamuthy M, Shukla HS: Predictive markers of response to neoadjuvant chemotherapy in breast cancer. Surg Oncol 2008, 17:30I-II.

25. Honkoop AH, Van Diest PJ, De Jong JS, Linn SC, Giaccone G, Hoekman K, Wagstaff J, Pinedo HM: Prognostic role of clinical, pathological and biological characteristics in patients with locally advanced breast cancer. $\mathrm{Br}$ J Cancer 1998, 77:62I-626.

26. Kariya S, Ogawa Y, Nishioka A, Moriki T, Ohnishi T, Ito S, Murata $Y$, Yoshida S: Relationship between hormonal receptors, HER-2, p53 protein, $\mathrm{Bcl}-2$, and MIB-I status and the antitumor effects of neoadjuvant anthracycline-based chemotherapy in invasive breast cancer patients. Radiation Medicine 2005, 23:189-94

27. Krajewski S, Krajewska M, Turner BC, Pratt C, Howard B, Zapata JM, Frenkel V, Robertson S, lonov Y, Yamamoto H, Perucho M, Takayama $S$, Reed JC: Prognostic significance of apoptosis regulators in breast cancer. Endocrine-Related Cancer 1999, 6:29-40.
28. Reed JC: Balancing cell life and death: Bax, apoptosis, and breast cancer. J Clinic Inves 1996, 97:2403-2404.

29. Stearns V, Singh B, Tsangaris T, Crawford JG, Novielli A, Ellis MJ, Isaacs C, Pennanen M, Tibery, Farhad A, Slack R, Hayes DF: A Prospective Randomized Pilot Study to Evaluate Predictors of Response in Serial Core Biopsies to Single Agent Neoadjuvant Doxorubicin or Paclitaxel for Patients with Locally Advanced Breast Cancer. Clinic Cancer Research 2003, 9:124-133.

30. Wolff AC, Davidson NE: Preoperative Therapy in Breast Cancer: Lessons from the Treatment of Locally Advanced Disease. The Oncologist 2002, 7:239-245.
Publish with Bio Med Central and every scientist can read your work free of charge

"BioMed Central will be the most significant development for disseminating the results of biomedical research in our lifetime. "

Sir Paul Nurse, Cancer Research UK

Your research papers will be:

- available free of charge to the entire biomedical community

- peer reviewed and published immediately upon acceptance

- cited in PubMed and archived on PubMed Central

- yours - you keep the copyright 